Article

\title{
Alginate Hydrogels Coated with Chitosan for Wound Dressing
}

\section{Maria Cristina Straccia ${ }^{1}$, Giovanna Gomez d'Ayala ${ }^{1}$, Ida Romano ${ }^{2}$, Adriana Oliva ${ }^{3}$ and Paola Laurienzo $1, *$}

1 Institute for Polymers, Composites and Biomaterials (IPCB), CNR, via Campi Flegrei 34, Pozzuoli 80078, Italy; E-Mails: mariacristina.straccia@ictp.cnr.it (M.C.S.); giovanna.gomez@ictp.cnr.it (G.G.A.)

2 Institute of Biomolecular Chemistry, CNR, via Campi Flegrei 34, Pozzuoli 80078, Italy; E-Mail: iromano@icb.cnr.it

3 Department of Biochemistry, Biophysics and General Pathology, Second University of Naples, via L. De Crecchio 7, Naples 80138, Italy; E-Mail: adriana.oliva@unina2.it

* Author to whom correspondence should be addressed; E-Mail: paola.laurienzo@ictp.cnr.it; Tel.: +39-81-867-5215; Fax: +39-81-867-5230.

Academic Editor: Alejandro M. Mayer

Received: 24 February 2015 / Accepted: 29 April 2015 / Published: 11 May 2015

\begin{abstract}
In this work, a coating of chitosan onto alginate hydrogels was realized using the water-soluble hydrochloride form of chitosan $(\mathrm{CH}-\mathrm{Cl})$, with the dual purpose of imparting antibacterial activity and delaying the release of hydrophilic molecules from the alginate matrix. Alginate hydrogels with different calcium contents were prepared by the internal setting method and coated by immersion in a $\mathrm{CH}-\mathrm{Cl}$ solution. Structural analysis by cryo-scanning electron microscopy was carried out to highlight morphological alterations due to the coating layer. Tests in vitro with human mesenchymal stromal cells (MSC) were assessed to check the absence of toxicity of $\mathrm{CH}-\mathrm{Cl}$. Swelling, stability in physiological solution and release characteristics using rhodamine $\mathrm{B}$ as the hydrophilic model drug were compared to those of relative uncoated hydrogels. Finally, antibacterial activity against Escherichia coli was tested. Results show that alginate hydrogels coated with chitosan hydrochloride described here can be proposed as a novel medicated dressing by associating intrinsic antimicrobial activity with improved sustained release characteristics.
\end{abstract}

Keywords: chitosan hydrochloride; alginate; hydrogels; antibacterial activity; sustained release; wound dressing 


\section{Introduction}

The local treatment of wounds is crucial to prevent infections, to control the removal of exudates and to create a moist environment to allow for skin healing [1]. Research is nowadays increasingly oriented towards "bioactive dressings". These dressings are made of materials that can play an active role in wound protection and healing. The first strategy consists of the application of skin substitutes, following a cell therapy approach [2]. A second strategy is the realization of "medicated dressings", able to release biomolecules in a sustained manner and to perform functions other than passive protection [3].

Due to their characteristics, hydrogels find application in wound dressing, especially in cases where a conventional dressing can be difficult to apply, as deep and irregular lesions. Hydrogels based on natural polysaccharides are highly hydrophilic and sometimes sensitive to enzymatic degradation [4-6]. In particular, alginates (Alg) are known for their ability to crosslink under mild conditions through a series of divalent cations. Alginates are a family of popular biocompatible polysaccharides extracted from brown algae [7]. Their hydrogels, in combination with other biopolymers or active agents, are used as dressing for wounds and burns, as they help to maintain an optimum moisture environment and cool temperature [8-10]. Alginate is approved for healthcare and present on the market with several trademarks. Another merit of alginate hydrogels is that they can be easily and quickly prepared at the time of need starting from sterilized solutions in a sterile area, making them appealing from a galenic, as well as an industrial point of view.

Alginate beads and hydrogels are stable in acidic media, whereas they easily swell and disintegrate in alkaline media and normal saline solution [11], conditions similar to those found in wound exudates. As calcium ions are being released by the ion exchange with sodium in the medium, electrostatic repulsion between the carboxylate anions further accelerates the swelling and erosion of alginate gels [12]. Moreover, on account of short time release in alkaline and neutral media, alginate is not an ideal material for sustained release. There were many attempts to control the disintegration of alginate-based drug delivery systems (DDS) and extend drug release characteristics through coating with polycationic polymers, such as poly(L-lysine) [13] or chitosan [14]. In contrast to alginate, which is polyanionic in nature, chitosan is a polycationic polysaccharide, derived from chitin $[15,16]$. Due to its gel-forming properties, it has been also employed in the design of DDS [17,18]. Complexation of alginate with chitosan reduces the porosity of the alginate gel and decreases the leakage of the encapsulated drugs [19-24]. In the case of wound dressing, encapsulated drugs are mainly antibiotics able to prevent or combat infections or growth factors to accelerate the healing process.

Nowadays, there are many concerns about the prolonged use of antibiotics, as required in the case of chronic wounds. The rise of bacterial resistance to antibiotics is well documented, both in the scientific literature and in the popular press. The World Health Organization recently described antimicrobial resistance as "a problem so serious that it threatens the achievements of modern medicine" [25]. Another concern is the safety of silver and titanium nanoparticles, often used in wound dressing for their antimicrobial activity [26,27]. Due to the small size, nanoparticles are easily absorbed into biological tissues and may interact with mitochondria, inducing structural damages, specifically in the liver cells [28]. In this regard, the use of materials with intrinsic antibacterial activity, avoiding the release of nanoscopic particles, may represent a valid alternative. As the antibacterial properties of chitosan are well documented in the literature [29,30], coating of alginate hydrogels with chitosan looks to be a 
possible strategy to realize intrinsic antimicrobial dressings with sustained release characteristics. Anyway, the difficulty in realizing alginate-chitosan hydrogels derives from their different $\mathrm{pH}$-dependent water solubilities. To overcome these drawbacks, several examples of the use of water-soluble chitosan derivatives are found in literature [31,32]. Nevertheless, chemical modification usually involves amines, with the loss of antibacterial activity.

In the present work, a coating of chitosan onto alginate hydrogels was realized using chitosan hydrochloride $(\mathrm{CH}-\mathrm{Cl})$, a protonated form of chitosan, with the dual purpose of imparting antibacterial activity and delaying the release of hydrophilic molecules from the alginate matrix. Chitosan hydrochloride was characterized by FTIR analysis. Alginate hydrogels with different calcium contents were prepared by the internal setting method and coated by immersion in a $\mathrm{CH}-\mathrm{Cl}$ solution. Structural analysis by cryo-scanning electron microscopy permits highlighting the morphological alterations due to the coating layer. Tests in vitro with human mesenchymal stromal cells (MSC) were assessed to check the absence of toxicity of CH-Cl. Swelling, stability in physiological solution and release characteristics using rhodamine $\mathrm{B}$ as the hydrophilic model drug were compared to those of relative uncoated hydrogels. Finally, the antibacterial activity against Escherichia coli was tested.

\section{Results and Discussion}

\subsection{Preparation of $\mathrm{CH}-\mathrm{Cl}$}

$\mathrm{CH}-\mathrm{Cl}$ is a charged form of chitosan, completely soluble in acid-free water [33]. It is known from the literature that the methodology employed here results in highly pure samples, which preserve an identical degree of deacetylation [34]. FTIR spectra of chitosan and $\mathrm{CH}-\mathrm{Cl}$ are shown in Figure 1. The appearance in the $\mathrm{CH}-\mathrm{Cl}$ spectrum of typical bands of symmetric and asymmetric stretching of ammonium N-H (1624 and $1518 \mathrm{~cm}^{-1}$, respectively) is evidence of the protonation of free amines.

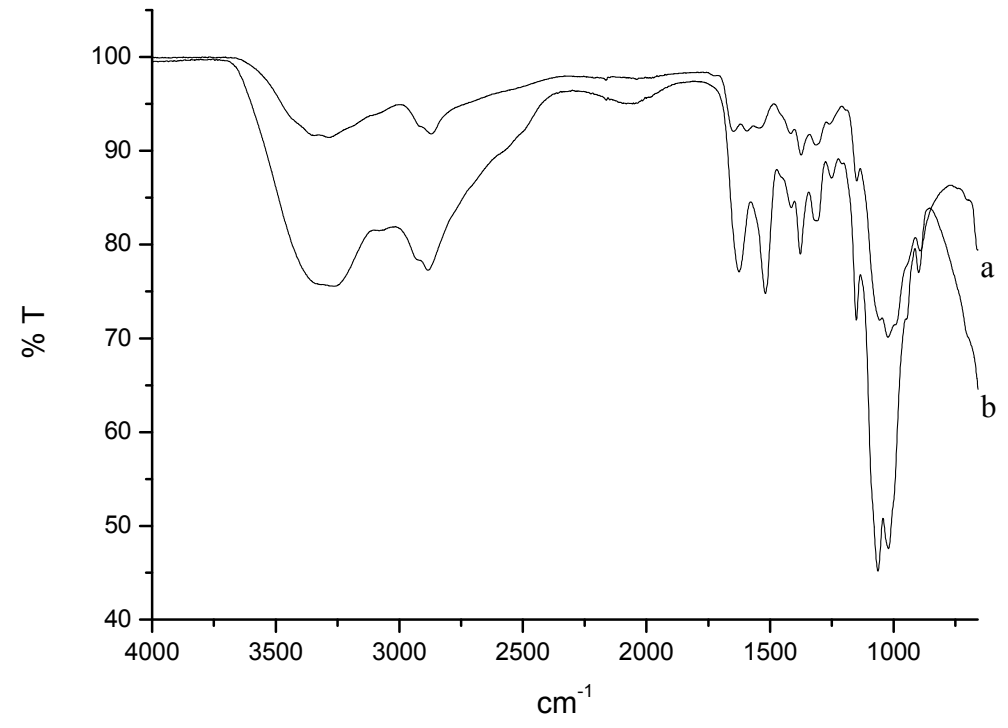

Figure 1. (a) FTIR spectrum of chitosan; (b) FTIR spectrum of chitosan hydrochloride (CH-Cl). 


\subsection{Preparation and Preliminary Characterization of Hydrogels}

Alginate hydrogels were prepared via internal gelation, using $\mathrm{CaCO}_{3}$ as the calcium source. This methodology allows one to obtain highly regular and homogeneous gels through a slow release of calcium ions. Different $\mathrm{CaCO}_{3}$ and $\mathrm{D}-(+)$-gluconate- $\delta$-lactone (GDL) amounts were used, as reported in Table 1.

Table 1. Composition and codes of hydrogels. GDL, D-(+)-gluconate- $\delta$-lactone; Alg, alginate.

\begin{tabular}{cccccc}
\hline $\mathbf{C a C O}_{\mathbf{3}}(\mathbf{m m o l})$ & $\mathbf{G D L}(\mathbf{m m o l})$ & $\mathbf{G D L}(\mathbf{m L})$ & $\left.\mathbf{A l g} / \mathbf{C a}^{\mathbf{2 +}} * \mathbf{( m o l} / \mathbf{m o l}\right)$ & $\mathbf{G D L} / \mathbf{C a C O}_{\mathbf{3}}(\mathbf{m o l} / \mathbf{m o l})$ & $\mathbf{C o d e}$ \\
\hline 0.999 & 0.999 & 17.8 & 5.155 & 1 & Alg1 \\
0.999 & 1.998 & 35.6 & 5.155 & 2 & Alg2 \\
1.998 & 1.998 & 35.6 & 2.577 & 1 & Alg3 \\
1.998 & 3.997 & 71.2 & 2.577 & 2 & Alg4 \\
\hline
\end{tabular}

* The reported data refer to $1 \mathrm{~g}$ of alginate $=0.0051 \mathrm{~mol}$ of repeating units.

The intrinsic features of hydrogels are strictly dependent on the relative concentrations of calcium carbonate and GDL. In particular, the molar ratio between GDL and calcium carbonate is crucial to obtain a complete salt dissolution and to determine the final gel $\mathrm{pH}$ (acid or neutral) [35,36], whereas the molar ratio between alginate repeating units and calcium ions regulates the number of carboxylates engaged in ionic interactions and, therefore, the crosslinking density of the resulting gel. Such characteristics are important for the final properties of the gel and for the interactions of alginate with chitosan hydrochloride, as will be discussed. Two GDL/CaCO 3 molar ratios were chosen, in order to obtain neutral or acid gels (1/1 and 2/1, respectively). Concerning the $\mathrm{Alg} / \mathrm{Ca}^{2+}$ ratio, a value close to the stoichiometric one or its half $(2.5 / 1$ and $5 / 1)$ was investigated.

Coating of alginate hydrogels was achieved simply by immersion in a $1 \%(w / w) \mathrm{CH}-\mathrm{Cl}$ water solution. The dipping time $(1 \mathrm{~h})$ and $\mathrm{CH}-\mathrm{Cl}$ concentration were established after several attempts. During immersion, shrinkage is observed for all hydrogels to a different extent. All coated hydrogels (c-Alg) retain transparency, a desirable feature for wound dressing to allow inspection of the injury bed. Furthermore, the hydrogels are harder and easier to handle upon coating.

Gelation time, bulk pH value and water content are reported in Table 2. Gelation time is mainly influenced by the calcium ion concentration, passing from a slow (around half an hour) to a fast (a few minutes) gelation when the amount of calcium carbonate is doubled. Of course, an excess of GDL ( $\mathrm{GDL} / \mathrm{CaCO}_{3}$ equal to two) further accelerates gelation as a consequence of two effects: faster calcium carbonate dissolution and partial acidification of carboxylate groups, which contribute to gelation via hydrogen bonding.

$\mathrm{pH}$ measurements evidence that hydrogels with a GDL/ $\mathrm{Ca}^{2+}$ molar ratio equal to one (Alg1 and $\mathrm{Alg} 3$ ) are basic, suggesting that calcium carbonate is not completely neutralized. When an excess of GDL is used (Alg2 and Alg4), the $\mathrm{pH}$ is lowered until neutral. After coating, a decrease of bulk pH values is generally observed, likely due to diffusion of $\mathrm{CH}-\mathrm{Cl}$ within the hydrogel during immersion. In particular, c-Alg2 and c-Alg4 show an acid pH value. 
Table 2. Gelation times, $\mathrm{pH}$ values and water content. c, coated.

\begin{tabular}{cccc}
\hline Sample & Gelation Time (min) & $\mathbf{p H}$ & Water Content (wt \%) \\
\hline Alg1 & $40 \pm 4$ & $8.34 \pm 0.14$ & $69.37 \pm 1.32$ \\
Alg2 & $28 \pm 3$ & $6.49 \pm 0.70$ & $68.61 \pm 1.15$ \\
Alg3 & $8 \pm 1$ & $7.24 \pm 0.83$ & $69.63 \pm 0.32$ \\
Alg4 & $5 \pm 1$ & $7.12 \pm 0.01$ & $68.76 \pm 0.84$ \\
c-Alg1 & $*$ & $7.53 \pm 0.54$ & $69.42 \pm 0.96$ \\
c-Alg2 & $*$ & $5.19 \pm 0.36$ & $67.70 \pm 0.82$ \\
c-Alg3 & $*$ & $7.34 \pm 0.81$ & $65.94 \pm 1.02$ \\
c-Alg4 & $*$ & $5.10 \pm 0.40$ & $53.51 \pm 0.64$ \\
\hline
\end{tabular}

* Gelation times are the same as the corresponding uncoated hydrogels.

The water content of Alg hydrogels is in line with what is reported in the literature for calcium alginate spray formulations [37]. Upon coating, a slight decrease is generally observed, likely due to the release of water associated with shrinking. This behavior is more pronounced in the case of c-Alg4, which visually shows greater shrinkage associated with volume reduction.

Figure 2 displays the gel homogeneity, estimated according to the literature [38]. The results show that, despite shrinking, the coating does not substantially influence the homogeneity of the gels.
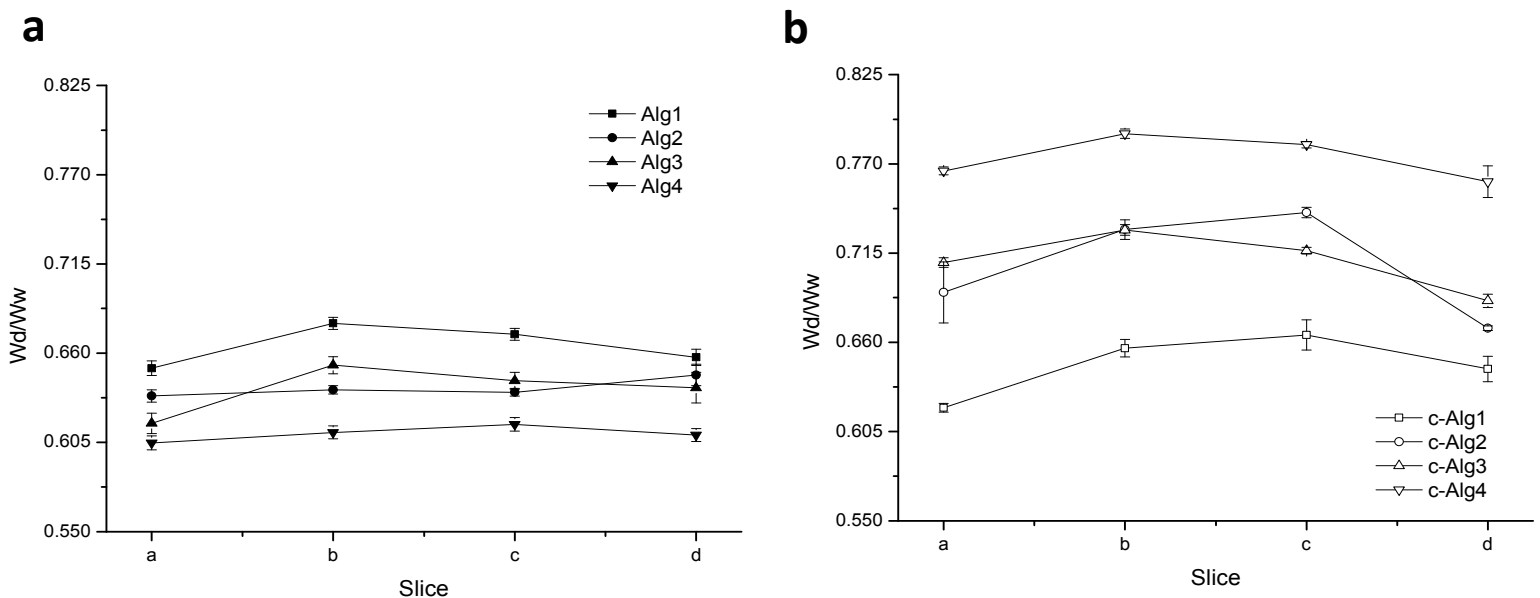

Figure 2. (a) Homogeneity profiles of Alg hydrogels; (b) homogeneity profiles of c-Alg hydrogels.

\subsection{Swelling}

The water uptake ability of hydrogels was monitored with time by weight determinations. Swelling kinetics curves are reported in Figure 3a,b. Alg hydrogels (Figure 3a) show a fast water uptake in the first two hours followed by a short plateau and a second phase in which swelling rapidly increases, perhaps due to incoming gel disintegration, until achievement of the equilibrium water content after around $8 \mathrm{~h}$. Swelling is generally related to crosslinking degree: hydrogels with a low crosslinking density are expected to have a large pore size and greater ability to swell, but ultimately may tend to dissolve. On the contrary, an increase of $\mathrm{Ca}^{2+}$ concentration or a $\mathrm{pH}$ decrease would limit swelling ability. Accordingly, swelling regularly decreases going from Alg1 to Alg4. Alg1 and Alg2 (low calcium content) reach over 400\% swelling, whereas Alg3 and Alg4 swell less (below 200\%). 

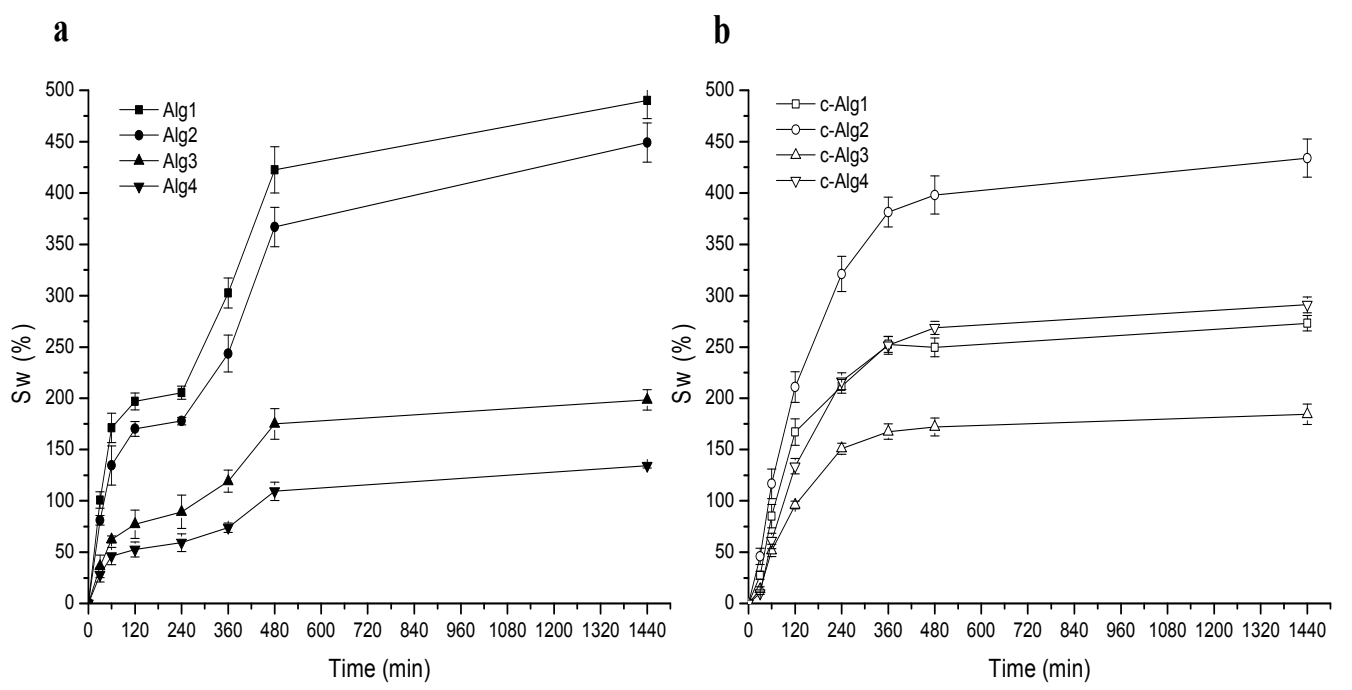

Figure 3. (a) Swelling curves of Alg hydrogels; (b) swelling curves of c-Alg hydrogels.

Swelling of c-Alg hydrogels increases regularly for all samples (Figure 3b), but the trend is not regular any more: whereas c-Alg2 preserves a high swelling percentage, close to that of Alg2, in the case of c-Alg1, swelling falls from $\sim 460 \%$ to $\sim 200 \%$ upon coating. This suggests that an alginate hydrogel with a low calcium concentration and besides being characterized by a basic $\mathrm{pH}$, such as Alg1, holds a greater amount of $\mathrm{CH}-\mathrm{Cl}$, due to the high number of free carboxylate groups available for ionic interactions with ammonium groups of $\mathrm{CH}-\mathrm{Cl}$, thus creating a tight coating layer in which ion pairs are uniformly distributed. It is known from the literature that ionic aggregates represent a barrier to water diffusion [39]; the strong swelling decrease of c-Alg1 is likely a consequence of the reduced water diffusion throughout the coating. Concerning c-Alg2, instead, the partial protonation of carboxylates, as evidenced by the $\mathrm{pH}$ value of $\mathrm{Alg} 2$, reduces the ionic interactions between alginate and chitosan hydrochloride; thus, $\mathrm{CH}-\mathrm{Cl}$ is just physically absorbed, and no significant variations of swelling properties are detected. Similarly, in the case of c-Alg3, carboxylate groups are saturated, due to the large excess of calcium ions, and no change of swelling occurs. On the opposite end, c-Alg4 shows higher swelling with respect to Alg4. This result is reasonably related to the re-uptake of water expelled during the coating process as a consequence of shrinkage (see Table 1 and the Discussion Section).

\subsection{Stability in Normal Saline Solution}

In order to verify the resistance of hydrogels to degradation in a medium close to the environment of the wound bed, a stability test was performed by immersion in normal saline solution for $24 \mathrm{~h}$. Curves relative to weight change percentage with time are shown in Figure $4 \mathrm{a}, \mathrm{b}$. It is important to underline that weight loss due to alginate chain dissolution is partially hidden by simultaneous swelling.

From a comparison, it is evident that coating induces an increase of stability: after initial swelling, Alg hydrogels undergo around a 10\% weight loss in eight hours, whereas c-Alg does not lose weight during the whole range of time. As can be seen, c-Alg has just a low weight loss of about $5 \%$ at first, attributed to the loss of $\mathrm{CH}-\mathrm{Cl}$ slightly absorbed onto the surface. Interactions between alginate and chitosan hydrochloride are not sensitive to sodium exchange, so the coating is stable, and disintegration 
of the hydrogel is delayed. It is also worth noticing that the Alg1 and Alg2 samples appear broken into several pieces just after two hours, while all other hydrogels preserve their physical integrity during $24 \mathrm{~h}$.
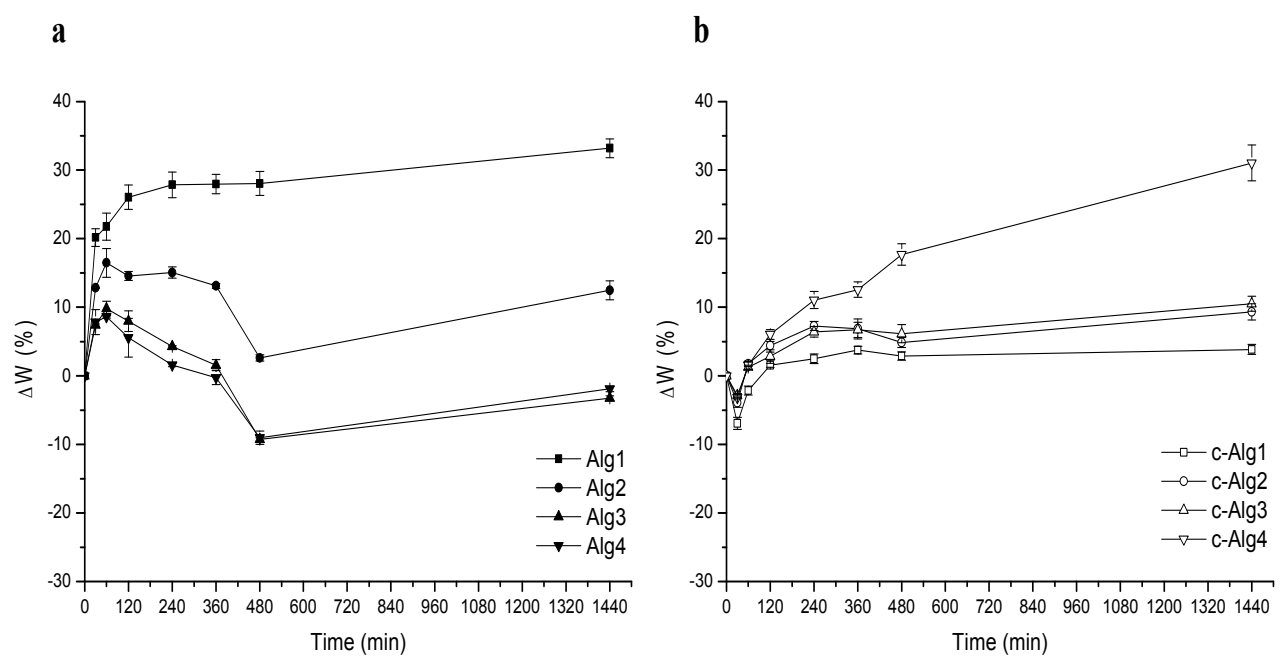

Figure 4. (a) Stability test in normal saline solution of Alg hydrogels; (b) stability test in normal saline solution of c-Alg hydrogels.

\subsection{Cryo-SEM Analysis}

Coated and uncoated hydrogels have been observed by cryo-SEM. Micrographs of Alg1 and c-Alg1 are reported in Figure 5a-f as an example. From a comparison between the outer surfaces of Alg1 (Figure 5a,b) and c-Alg1 (Figure 5d,e), it seems that in the last case, the surface is more regular and compact, whereas Alg1 shows an irregular surface, with the presence of numerous depressions, likely due to water evaporation. This evidence suggests a reduction in water loss from the coated hydrogel. Low-temperature fracture allows the exposure of the internal structure; the pore dimension can be roughly estimated in the range of 10-20 $\mu \mathrm{m}$ (Figure 5c,f). The morphology of the internal structure is not altered by the presence of the coating layer.
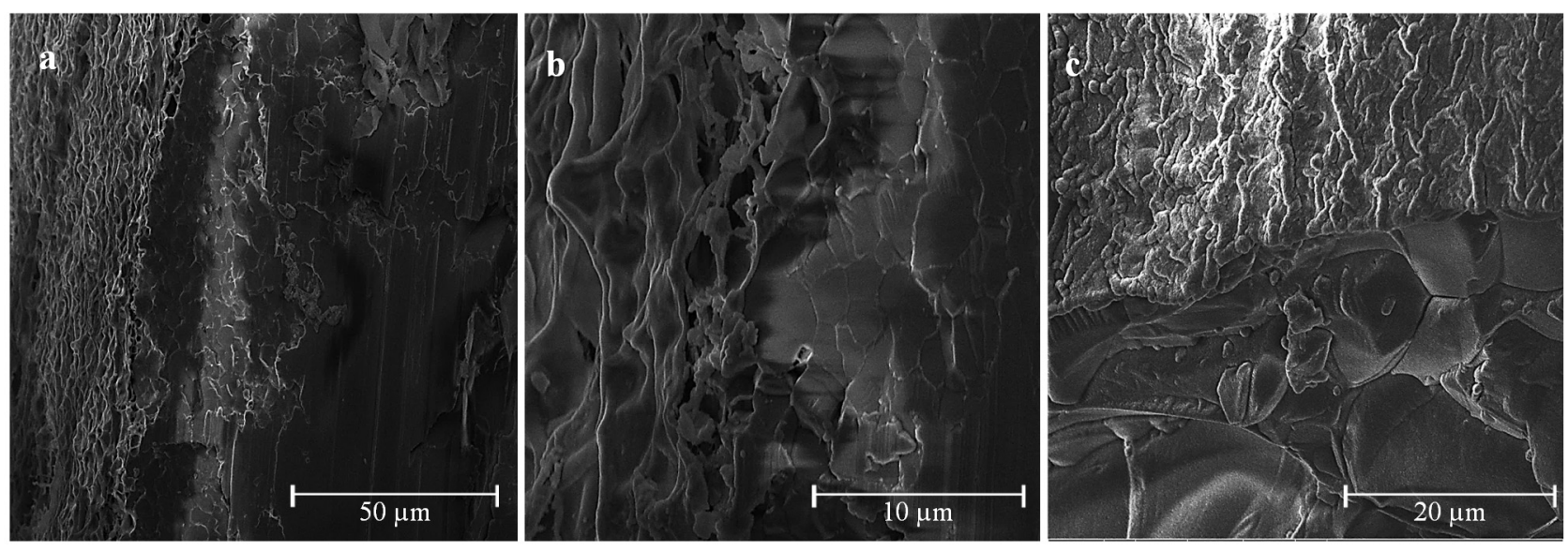

Figure 5. Cont. 

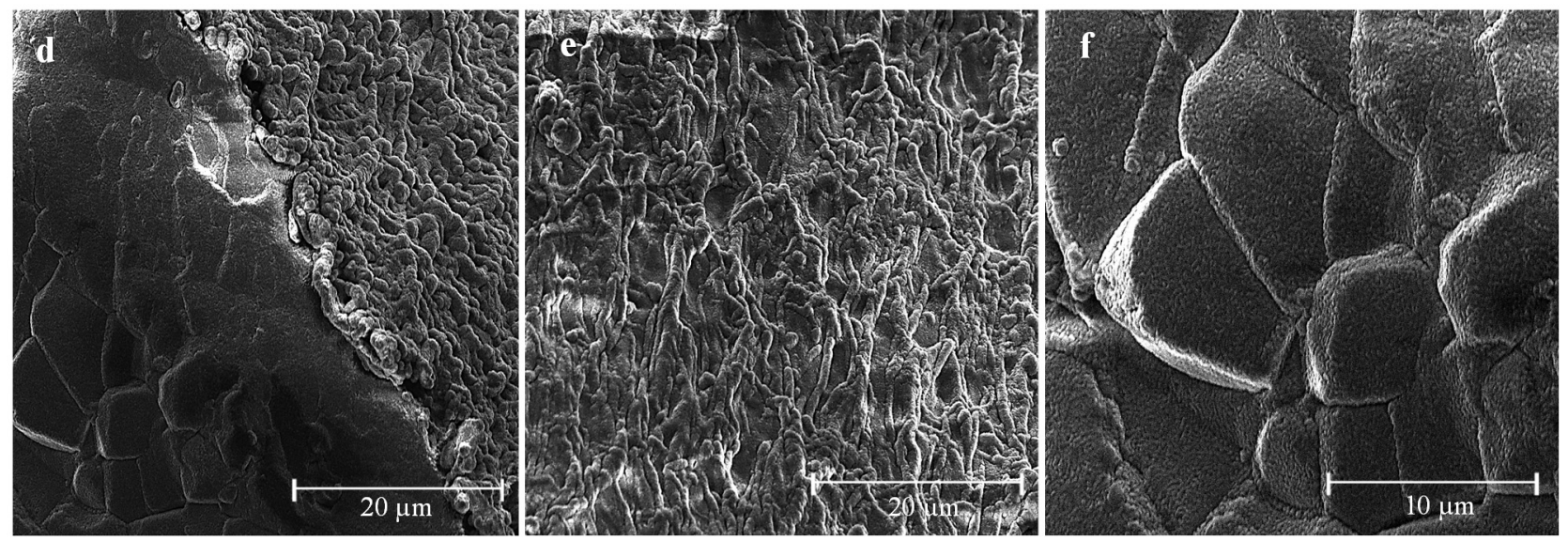

Figure 5. (a-c) Cryo-SEM images of Alg1; (d-f) cryo-SEM images of c-Alg1.

\subsection{Release Study}

In order to verify the effect of coating on the release properties, rhodamine $\mathrm{B}(\mathrm{RhB})$ was chosen as a model of a low molecular weight hydrophilic drug and encapsulated within the hydrogel. To evaluate the release kinetics in physiological conditions, hydrogels were placed on a porous glass set in order to be in contact with PBS medium ( $\mathrm{pH}$ 7.4) only by the lower surface (Figure 6). This equipment was designed to mimic exudate penetration into the hydrogel from the wound bed. The release profiles of Alg hydrogels compared to coated hydrogels are reported in Figure 7. As can be seen, c-Alg always exhibits a lower release rate with respect to the relative uncoated hydrogels. After eight hours, Alg hydrogels released about $70 \%-80 \%$ of their content, whereas c-Alg hydrogels released only about $60 \%-50 \%$. The different rate of release can be easily related to the presence of a coating layer on the hydrogel surface. Release from hydrogels is dependent on the diffusion of drug molecules throughout the alginate matrix; the presence of a coating layer acts as a barrier that delays diffusion and, hence, slows down release.

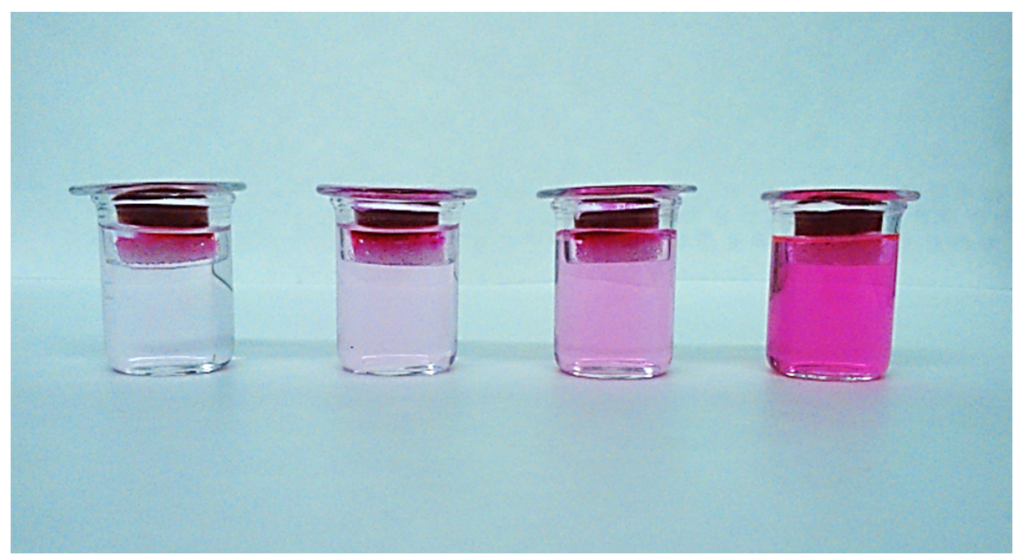

Figure 6. Equipment employed to study the release of rhodamine B in physiological conditions. The photo refers to different release times. 
a

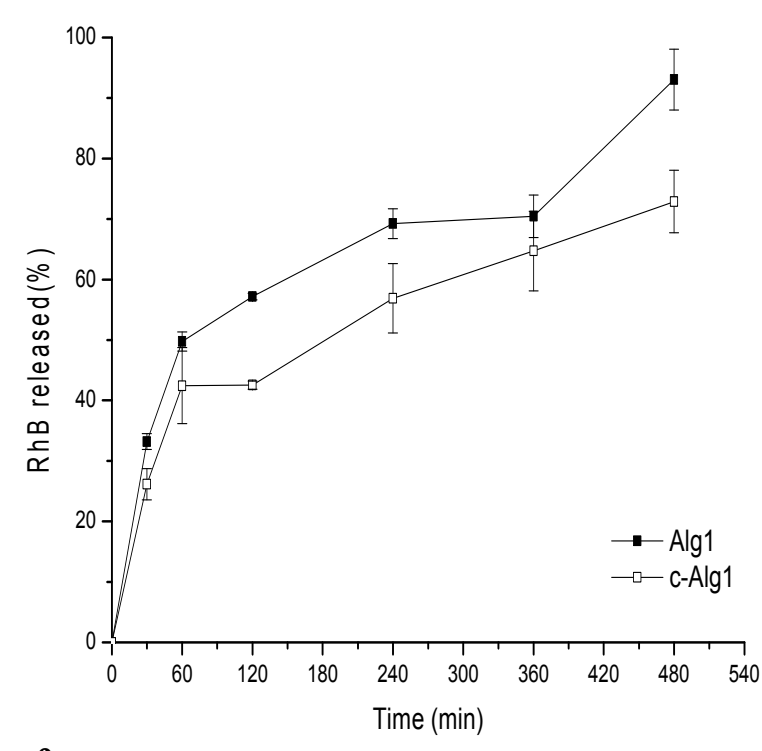

C

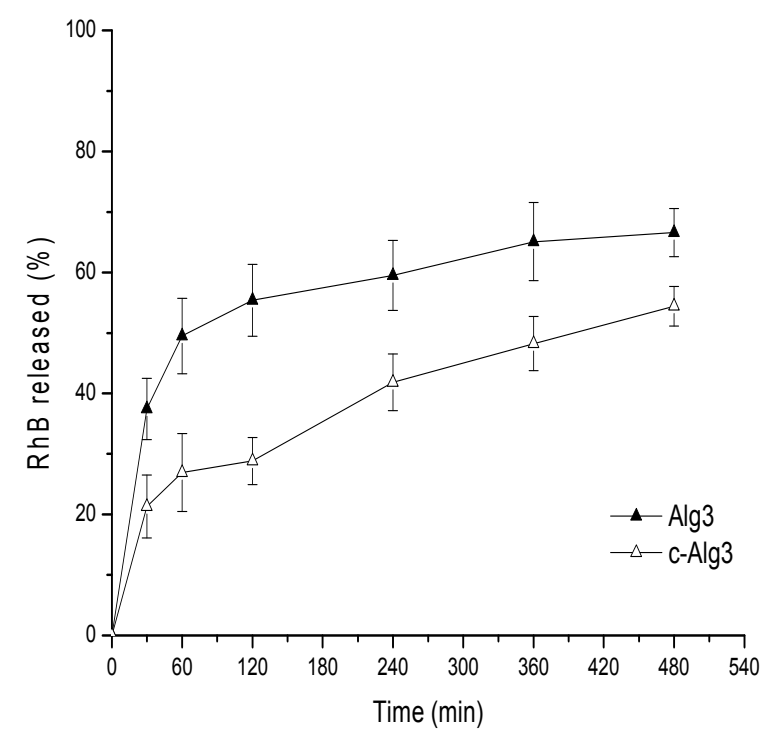

b

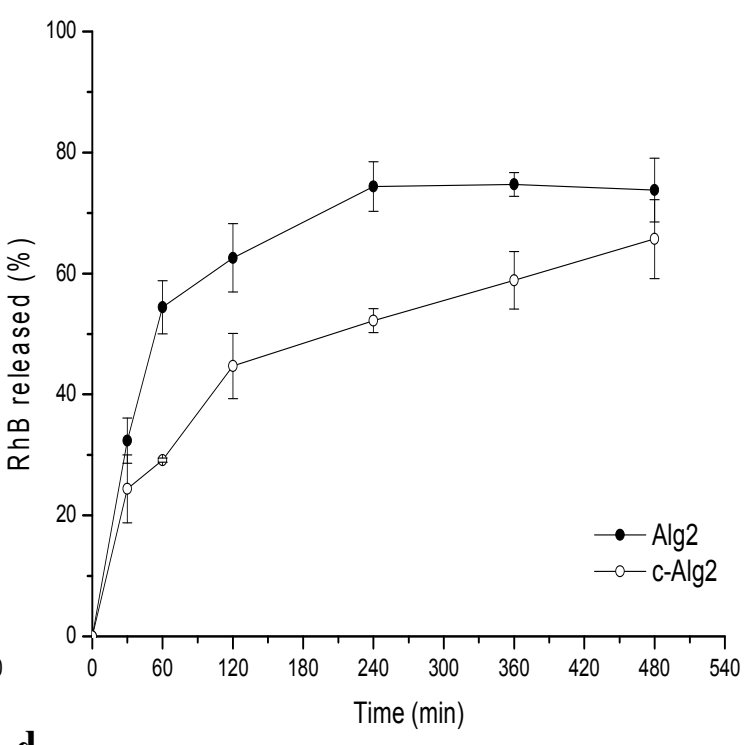

d

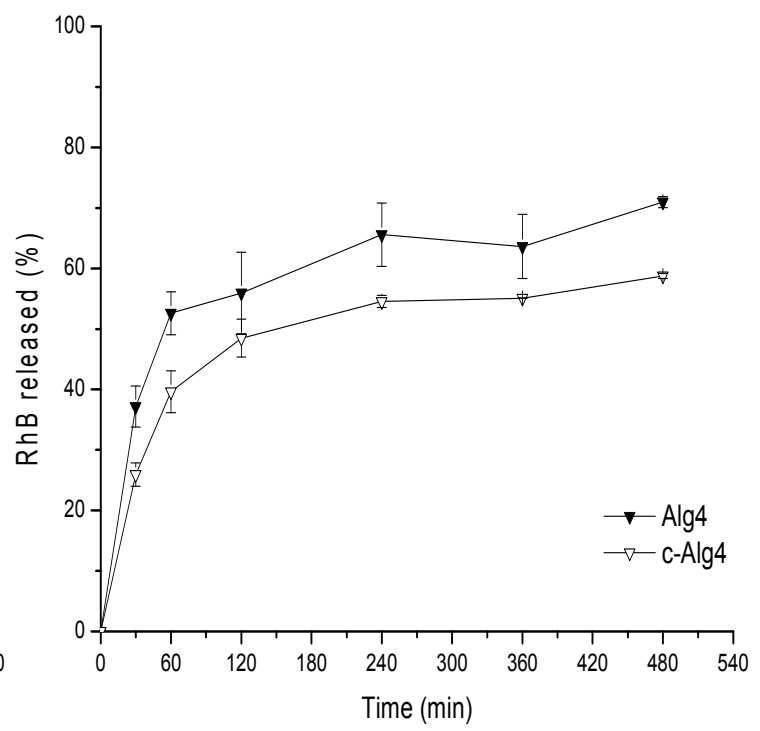

Figure 7. Release profiles of RhB from: (a) Alg1 and c-Alg1; (b) Alg2 and c-Alg2; (c) Alg3 and c-Alg3; (d) Alg4 and c-Alg4.

\subsection{Cytotoxicity Test}

A cytotoxicity test to analyze the effects of $\mathrm{CH}-\mathrm{Cl}$ solution on mesenchymal stromal cells (MSC) was performed. Cells were treated for seven days with culture medium (control) or with culture medium added with $0.1 \%(w / v)$ solution of $\mathrm{CH}-\mathrm{Cl}$. MSC were examined every day for one week to observe any possible appearance of cytotoxicity signs, such as morphologic changes, cellular lysis areas or cell death. Figure 8 shows the microscopic images of cells cultured for one week in control medium $(\mathrm{a}, \mathrm{b})$ and in $0.1 \% \mathrm{CH}-\mathrm{Cl}$ medium (c,d) after crystal violet staining. It appears evident that in both cases (control and treated MSC), the morphology was substantially unmodified; most important, growth was not inhibited, and cells were able to reach confluence to the same extent as the controls. Anyway, it has to be underlined that this test represents only the first, pivotal approach in the evaluation of biocompatibility, which 
allows one to exclude an acute cytotoxicity. Other tests, namely sensitization and irritation tests, will be necessary to confirm the absence of adverse effects on living tissues.
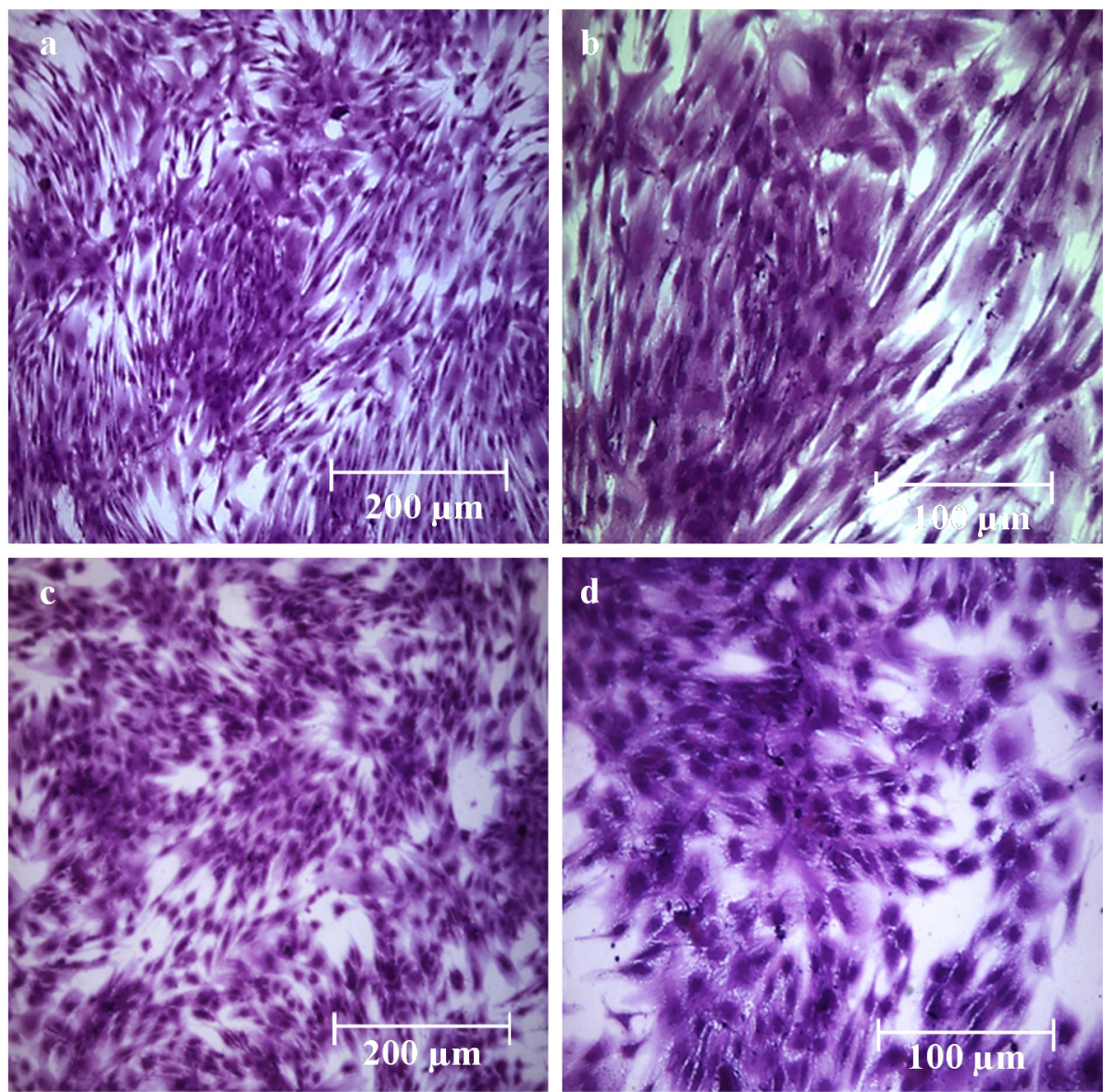

Figure 8. (a,b) Microscopic images of MSC cultured for seven days in control culture medium after crystal violet staining; (c,d) microscopic images of MSC cultured for seven days in $0.1 \% \mathrm{CH}-\mathrm{Cl}$ culture medium after crystal violet staining.

\subsection{Antibacterial Activity}

The antibacterial activity of $\mathrm{CH}-\mathrm{Cl}$ and c-Alg was tested against the Escherichia coli wild-type strain, the second most common single pathogen involved in postoperative wound infections [40]. To test the activity of $\mathrm{CH}-\mathrm{Cl}$ as an antimicrobial agent, $\mathrm{MIC}$ and minimum bactericidal concentration (MBC) were determined. MIC and MBC define the antibacterial efficiency of an antimicrobial agent in terms of the concentration at which it will inhibit growth (MIC) or completely kill (MBC) $1 \times 10^{6}$ challenge microorganisms during $24 \mathrm{~h}$ of incubation. Both $\mathrm{MIC}$ and $\mathrm{MBC}$ were found to fall at a 1:32 dilution, corresponding to $0.31 \mathrm{mg} / \mathrm{mL}$ of $\mathrm{CH}-\mathrm{Cl}$.

Preliminary tests based on the solid agar medium contact method carried out on hydrogels evidenced the presence of an inhibition zone around the contact area in the case of coated hydrogels, completely absent in uncoated hydrogels. Figure 9a,b shows photos of Escherichia coli growing on agar plates in contact with Alg1 and c-Alg1 hydrogels as an example. No significant differences were noted among the coated hydrogels (c-Alg1, c-Alg2, c-Alg3 and c-Alg4). 


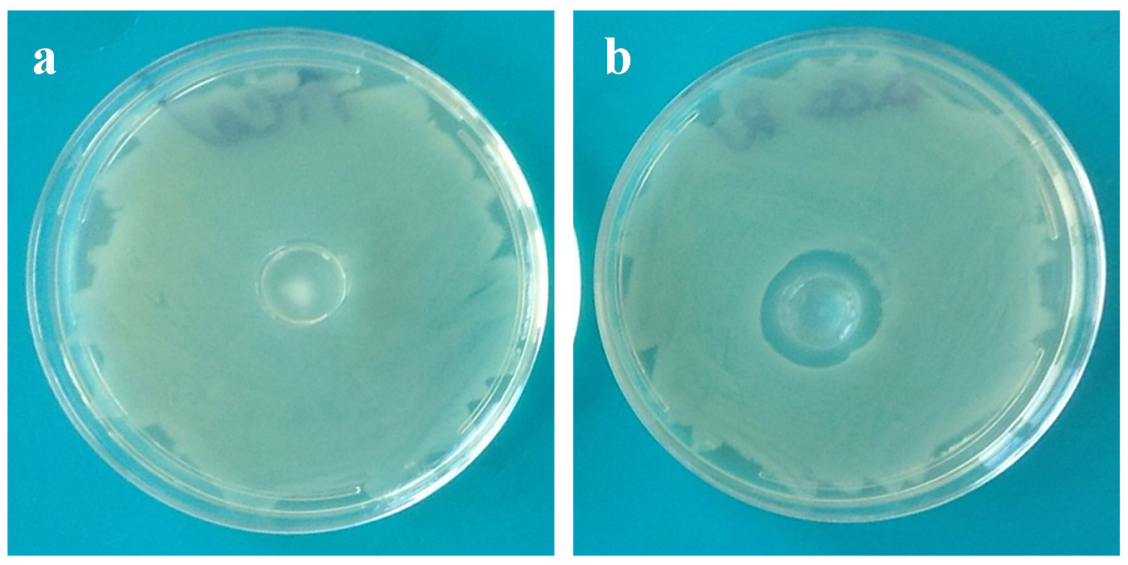

Figure 9. (a) Optical photo of $E$. coli growing on agar in contact with Alg1; (b) optical photo of $E$. coli growing on agar in contact with c-Alg1.

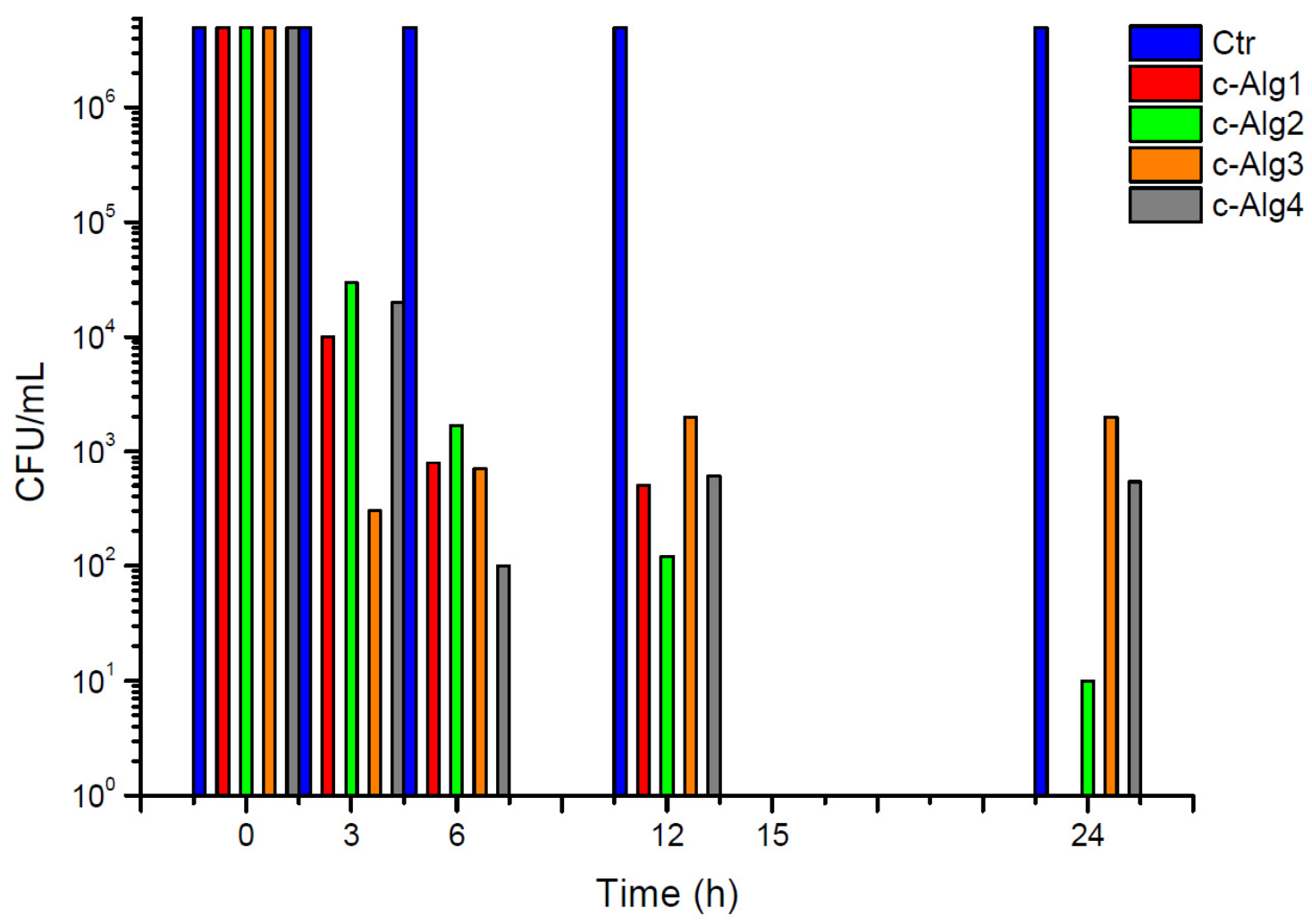

Figure 10. Antimicrobial activity kinetics of c-Alg hydrogels against E. coli.

The kinetics of antimicrobial activity against Escherichia coli of coated hydrogels is reported in Figure 10. Uncoated hydrogels were tested as the negative control (Ctr). Results show that all c-Alg cause a bacterial inactivation higher than $99 \%$ already after $3 \mathrm{~h}$ of contact, and a complete killing of bacteria was reached after $24 \mathrm{~h}$ in the case of c-Alg1. The concentration of $\mathrm{CH}-\mathrm{Cl}$ is likely equal or higher than the MIC and MBC values in all coated hydrogels. The slight difference in bactericidal activity among hydrogels is attributed to the different degree of interaction between $\mathrm{CH}-\mathrm{Cl}$ and alginate in the various hydrogels, as previously discussed (e.g., swelling behavior, see Section 2.2). Alginate hydrogel with the highest concentration of free carboxylate groups (Alg1) binds more $\mathrm{CH}-\mathrm{Cl}$ via ionic interactions with ammonium groups, forming a denser layer coating on the surface. As a consequence, 
bacterial killing is complete, whereas surviving bacterial cells (less than 1\%) are found with the other hydrogels. Furthermore, in the case of c-Alg3 and c-Alg4, the activity stops after $6 \mathrm{~h}$, as no further reduction is detected after this time. Once again, the occurrence of antibacterial activity can be related to the interaction of alginate with $\mathrm{CH}-\mathrm{Cl}$. In fact, in the case of hydrogels with high calcium content (c-Alg3 and c-Alg4), most of the carboxylates groups of alginate are engaged in calcium ion chelation and not available for interactions with ammonium groups, reducing the coating layer.

\section{Experimental Section}

\subsection{Materials}

Pharmaceutical-grade alginic acid sodium salt (Alg), extracted from Laminaria hyperborean (viscosity $360 \mathrm{mPa} \cdot \mathrm{s}, 1 \% \mathrm{w} / v$ water solution; mannuronic and guluronic acids: ratio 1.8:2.2), was supplied by Farmalabor (Canosa di Puglia, Brindisi, Italy). Chitosan $(\mathrm{CH})$ extracted from crab shells (high molecular weight; viscosity $400 \mathrm{mPa} \cdot \mathrm{s}, 1 \%$ acetic acid $\left(20{ }^{\circ} \mathrm{C}\right) ; 80 \%$ deacetylation degree), calcium carbonate, D- $(+)$-glucono- $\delta$-lactone $(\mathrm{GDL})$, rhodamine $\mathrm{B}(\mathrm{RhB})$ and sodium phosphate monobasic monohydrate $\left(\mathrm{Na}_{2} \mathrm{HPO}_{4} \cdot \mathrm{H}_{2} \mathrm{O}\right)$ were purchased from Sigma Aldrich (Milan, Italy). Streptomycin sulfate was supplied by Applichem (Darmstadt, Germany). Potassium chloride, sodium bicarbonate, calcium chloride hexahydrate and sodium chloride were purchased from J.T. Baker (Avantor Performance Materials, Milan, Italy). Yeast extract, tryptone and agar, used as the culture medium, were purchased from Oxoid, England. Opti-MEM was purchased from Life technologies Italia (Monza, Italy).

\subsection{Preparation of Chitosan Hydrochloride}

Water-soluble chitosan hydrochloride $(\mathrm{CH}-\mathrm{Cl})$ was prepared following literature reports [34]. Briefly, a solution of chitosan $(1 \mathrm{~g} / 100 \mathrm{~mL}, 1 \%$ acetic acid) was filtered and dialyzed against $\mathrm{NaCl} 0.4 \mathrm{~mol} / \mathrm{L}$ for eight days. $\mathrm{NaCl}$ solution was then replaced by freshly-distilled water, and the dialysis continued for another two days. $\mathrm{CH}-\mathrm{Cl}$ was finally recovered by freeze-drying in the form of white powder.

\subsection{Preparation of Alginate Hydrogels by Internal Setting Method}

Alginate hydrogels were prepared using the internal setting method [35,36]. Fine suspensions of calcium carbonate in distilled water $(0.1 \%$ or $0.2 \% \mathrm{w} / \mathrm{v})$ were obtained by sonication (Vibracell VC 505, Sonics, Newton, CT, USA; $500 \mathrm{~Hz}, 50 \%$ amplitude, $10 \mathrm{~min}$ ). One gram of sodium alginate was dissolved in $100 \mathrm{~mL}$ of suspension under stirring, then a chosen amount of $1 \% w / v$ GDL solution was added. Five milliliters of each final alginate solution were immediately poured into a petri dish $(3.6 \mathrm{~cm}$ diameter) and left for $24 \mathrm{~h}$ in air at room temperature to complete gelation. Hydrogels were washed in water by quick immersion and briefly blotted on paper before characterization.

\subsection{Preparation of Coated Hydrogels}

Samples coated with $\mathrm{CH}-\mathrm{Cl}$ were prepared by immersion in $\mathrm{CH}-\mathrm{Cl}$ solution. Freshly-prepared alginate hydrogels (1.9 cm diameter, corresponding to $1 \mathrm{~mL}$ of alginate solution) were embedded in 
$10 \mathrm{~mL}$ of $\mathrm{CH}-\mathrm{Cl}$ solution $(1 \mathrm{~g} / 100 \mathrm{~mL}$, water) for $60 \mathrm{~min}$ under mild stirring. The coated hydrogels were carefully recovered with a strainer and withdrawn by briefly blotting on paper.

\subsection{FTIR Analysis}

FTIR spectra were obtained in the attenuated total reflection mode (ATR) using a Perkin-Elmer spectrometer (Norwalk, CT, USA) equipped with universal-ATR accessory, fitted with a diamond optical element and $\mathrm{ZnSe}$ focusing elements. The apparatus operates with a single reflection at an incident angle of $45^{\circ}$. The analysis was carried out on powders at room temperature and ambient humidity. Spectra were acquired between 4000 and $400 \mathrm{~cm}^{-1}$ with a spectral resolution of $4 \mathrm{~cm}^{-1}$ and 32 scans collected.

\subsection{Gelation Time}

Gelation time, defined as the time between the addition of GDL and the formation of a fixed gel, was assessed using a method adapted from the literature [38]. The petri dish containing the gelling solution was periodically tilted during the gelation process. Gel was said to be formed when there was no longer flowing when the dish was kept at an angle of $45^{\circ}$ for more than $30 \mathrm{~s}$.

\section{7. $\mathrm{pH}$ Measurements}

Bulk pH determinations were carried out by a CRISON 507 pH-meter (CRISON, Barcelona, Spain) equipped with type 52-00 electrodes and a probe tip 52-32 for penetration analysis.

\subsection{Water Content}

Water content (W) of hydrogels was calculated as a percentage by the ratio between the wet gel weight and the dry gel weight (after drying at $40{ }^{\circ} \mathrm{C}$ in air until constant weight). Measurements were performed in triplicate.

\subsection{Homogeneity}

The homogeneity of alginate gels was estimated according to the literature [38]. Hydrogels were cut into four slices of equal dimension $(\mathrm{a}-\mathrm{d})$. Each slice was weighed, then dried to constant weight and reweighed. The dry to wet weight ratio $\left(\mathrm{W}_{\mathrm{d}} / \mathrm{W}_{\mathrm{w}}\right)$ of slices provides an indication of the homogeneity. A homogeneous gel will have a consistent dry to wet weight ratio across its constituent slices. Data are the mean of three measurements for each sample.

\subsection{Swelling}

Swelling was determined gravimetrically by monitoring the water up-take with time. Hydrogels were immersed in distilled water, withdrawn at different times and weighed after blotting on paper. For each sample, the measurements were performed in triplicate, and average data were used for the calculations. 


\subsection{Stability Test}

A degradation test in normal saline solution $(\mathrm{NaCl} 0.9 \%, w / v)$ was achieved in order to get information about the stability of hydrogels when in contact with physiological fluids. Hydrogels were weighed and immersed in $100 \mathrm{~mL}$ of saline solution at room temperature. At set time intervals, the samples were withdrawn, briefly blotted on filter paper and weighed. Stability was expressed as weight variation percentage $(\Delta \mathrm{W} \%)$. All measurements were performed in triplicate, and average data were reported.

\subsection{Cryo-SEM Analysis}

The morphology of coated hydrogels was examined through cryo-scanning electron microscopy (cryo-SEM) using a cryo-system Gatan Alto 1000E (Gatan, Pleasanton, CA, USA) installed on a FEI Quanta 200 FEG SEM (FEI, Eindhoven, The Netherlands). The sample was placed on the holder, mounted on the cryo-transfer rod, slam-frozen in nitrogen slush and transferred to the cryo-chamber, where it was cryo-fractured and sputter coated with gold/palladium. The sample was finally moved to the SEM chamber where either fracture or top surfaces were observed at $-140{ }^{\circ} \mathrm{C}$, using an acceleration voltage of $5-10 \mathrm{kV}$.

\subsection{Release Study}

Alg and c-Alg hydrogels (1.9 cm diameter), prepared as previously described (Section 2.3), were loaded with $\mathrm{RhB}$ by dissolving $10 \mathrm{mg}$ of alginate in $1 \mathrm{~mL}$ of $\mathrm{RhB}$ aqueous solution of different concentrations, in order to obtain in each gel a final $\mathrm{RhB}$ concentration of $13.05 \mu \mathrm{M}$. Release studies were performed using a modified Enslin apparatus, designed to mimic the wound bed [41]. Twelve milliliters of phosphate buffer saline solution (PBS; NaCl $120 \mathrm{mM}, \mathrm{KCl} 2.7 \mathrm{mM}, \mathrm{Na}_{2} \mathrm{HPO}_{4} 10 \mathrm{mM}$, $\mathrm{pH}$ 7.4) were in contact with the hydrogels only by their lower surface. At set times, $1.0 \mathrm{~mL}$ of buffer medium was withdrawn, replaced by the same amount of fresh PBS and analyzed by UV spectroscopic analysis. Release was followed for $8 \mathrm{~h}$. The RhB\% released was calculated using a 10-point calibration curve $\left(R^{2}=0.9993\right)$ in the concentration range 13.05-1.30 $\mu \mathrm{M}$. Experiments were performed in triplicate.

\subsection{Cytotoxicity Test}

In order to exclude the cytotoxic effects of chitosan hydrochloride, an assay was performed on mesenchymal stromal cells (MSC) obtained from normal human bone marrow, as previously described [42]. Cells were seeded in multi-well plates at a density of 20,000/ $\mathrm{cm}^{2}$ and treated with culture medium (control) or with culture medium added with $0.1 \%(w / v)$ solution of $\mathrm{CH}-\mathrm{Cl}$. The test was performed in triplicate. MSC were grown for one week renewing the medium every second day and observed daily by optical microscopy. Finally, control and treated MSC were stained with crystal violet and observed with a Workstation Leica DMI6000 microscope (Leica Microsystems GmbH, Wetzlar, Germany). Images were acquired using a digital camera Leica DFC 340FX (Leica Microsystems GmbH, Wetzlar, Germany) and analyzed by LAS AF 2.2.0 software. 


\subsection{Antibacterial Activity}

Antibacterial activity of CH-Cl and c-Alg hydrogels was tested against Escherichia coli (DSM 498), purchased from Deutsche Samlug von Mikroorganismen und Zellkulturen GmbH (DSMZ, Braunschweig, Germany). Growth of bacterial strain occurs on sterile Luria-Bertani (LB) medium (10 g/L tryptone, $5 \mathrm{~g} / \mathrm{L}$ yeast extract, $10 \mathrm{~g} / \mathrm{L} \mathrm{NaCl}$ distilled water) in an aerated incubator at $37{ }^{\circ} \mathrm{C}$, $120 \mathrm{rpm}$, for 18-24 h. Bacterial growth was verified measuring the optical density of bacteria at $600 \mathrm{~nm}$ $\left(\mathrm{OD}_{600}\right)$ by means of a spectrophotometer. After growth and harvesting, bacterial cells were washed twice with sterile Ringer solution (RS), a neutral buffer saline solution $(0.150 \mathrm{~g} \mathrm{KCl}, 2.25 \mathrm{~g} \mathrm{NaCl}$, $0.05 \mathrm{~g} \mathrm{NaHCO}_{3}$ and $0.12 \mathrm{~g} \mathrm{CaCl}_{2}$ per liter of distilled water, $\left.\mathrm{pH} 7.0\right)$ and then re-suspended in $\mathrm{RS}$ up to an absorbance of $0.250 \pm 0.01$, which corresponds to a concentration of approximately $1.5-3.0 \times 10^{8}$ colony-forming units per milliliter $(\mathrm{CFU} / \mathrm{mL})$. A second dilution was carried out to obtain working bacterial suspensions of about $10^{6} \mathrm{CFU} / \mathrm{mL}$.

The minimum inhibitory concentration (MIC) of $\mathrm{CH}-\mathrm{Cl}$, defined as the lowest concentration of the antimicrobial agent that inhibits the visible growth of the test microorganism, was determined using the broth dilution methods $[43,44]$. $\mathrm{CH}-\mathrm{Cl}$ solution $(10 \mathrm{mg} / \mathrm{mL}$, water $)$ was serially diluted with LB medium: $10 \mathrm{~mL}$ of $\mathrm{CH}-\mathrm{Cl}$ solution were added to $10 \mathrm{~mL}$ of $\mathrm{LB}$ and mixed by vortexing for $1 \mathrm{~min}$; successive dilutions were repeated in order to obtain a concentration range spanning from 0.078 to $10 \mathrm{mg} / \mathrm{mL}$. Ten milliliters of each diluted $\mathrm{CH}-\mathrm{Cl}$ solution were inoculated with $100 \mu \mathrm{L}$ of $10^{8} \mathrm{CFU} / \mathrm{mL}$ microbial suspension of Escherichia coli. The inoculum assay and streptomycin sulfate $(15 \mu \mathrm{g} / \mathrm{mL})$ were evaluated as negative and positive controls, respectively; the optical density of bacteria after $24 \mathrm{~h}$ was assessed.

The minimum bactericidal concentration (MBC) was measured following MIC determination. One hundred microliters of each of the two dilution suspensions preceding the MIC dilution were plated onto LB medium agar $1.8 \%$ and incubated at $37{ }^{\circ} \mathrm{C}$ overnight. The highest dilution (and conversely, the lowest concentration) that resulted in a $99.9 \%$ reduction in bacterial cells number was recorded as the MBC.

Each measurement was replicated three times.

Preliminary screening of the antimicrobial properties of c-Alg hydrogels $(1.9 \mathrm{~cm}$ diameter $)$ was carried out by the solid phase contact method on an LB agar medium plate (1.5\% agar) in petri dishes. Hydrogels were prepared in sterile conditions. Working bacterial suspensions $(0.1 \mathrm{~mL})$ were spread onto the solid surface of the medium agar plate, then hydrogels were put on it, and the plates were incubated at $37^{\circ} \mathrm{C}$ for $24 \mathrm{~h}$. After incubation, the inhibition areas were evaluated. Alg hydrogels were tested as the negative control.

The kinetics of killing of c-Alg1, c-Alg2, c-Alg3 and c-Alg4 (1.9 cm diameter) was determined by measuring bacteria logarithmic reduction as a function of time. Hydrogels were kept in contact with $10 \mathrm{~mL}$ of working bacterial suspension at room temperature on a wrist-action shaker (ASTM standard Test Method E 2149-01). Then, $0.1 \mathrm{~mL}$ of working solution were used to prepare decimal dilutions, which were plated onto the LB solid medium agar plate. Plates were incubated at $37{ }^{\circ} \mathrm{C}$ for $24 \mathrm{~h}$. After $0 \mathrm{~h}\left(t_{0}\right), 3 \mathrm{~h}\left(t_{3}\right), 6 \mathrm{~h}\left(t_{6}\right), 12 \mathrm{~h}\left(t_{12}\right)$ and $24 \mathrm{~h}\left(t_{24}\right)$ of contact time, surviving cells were evaluated by the standard plate count method, and inactivation tests were performed in duplicate. The inoculum assay and Alg hydrogels were used as the negative control, while streptomycin sulfate $(15 \mu \mathrm{g} / \mathrm{mL})$ was tested as the positive control. The average colony count of duplicate plates was used to calculate the CFU/mL. Tests were performed three times. 
The percentage reduction was calculated by the following equation:

$$
\text { Reduction \% (CFU mL } \left.{ }^{-1}\right)=\left(\frac{B-A}{B}\right) \times 100
$$

where $A=$ bacterial concentration after a specific contact time and $B=$ bacterial concentration at $t_{0}$ contact time.

\section{Conclusions}

Alginate chitosan-coated hydrogels were prepared by using water-soluble chitosan hydrochloride. The internal gelation setting method was used for the realization of hydrogels. Coated hydrogels retain good homogeneity and high water content, whereas a decrease of bulk $\mathrm{pH}$ was detected. Overall, hydrogels were found to have a water uptake weight percentage ranging from 450 to 200, which would prevent the wound bed from accumulating exudates and, at the same time, protect it from excessive dehydration. Stability in normal saline solution increases upon coating. Cryo-SEM analysis highlighted a more regular and compact surface on coated hydrogels, while the internal morphology was not altered. Coated hydrogels exhibit antibacterial activity against Escherichia Coli, and cytotoxicity tests demonstrate that chitosan hydrochloride does not elicit any acute toxic effects on mesenchymal stromal cells. Release studies using rhodamine B as a model of a low molecular weight hydrophilic drug show that the coating induces a decrease in the release kinetics.

To summarize, in this work, novel hydrogels based on alginate and chitosan hydrochloride have been investigated in order to explore their potential application as novel medicated dressings by associating intrinsic antimicrobial activity with improved sustained release characteristics.

\section{Acknowledgments}

The authors gratefully acknowledge the project "High-tech devices for biomedical applications" (acronym: DIATEME) in the frame of the National Operative Program (PON 2007-2013), for financial support. The authors wish to thank Cristina Del Barone from Laboratory of Electron Microscopy "LaMEST" of IPCB, CNR for technical assistance with the cryo-SEM analysis. The authors are also indebted to Roberta Imperatore (Institute of Applied Sciences and Intelligent Systems, CNR) for assistance with the optical microscopy.

\section{Author Contributions}

Conceived and designed the experiments: GGA AO PL. Performed the experiments: MCS, IR. Analyzed the data: MCS, GGA, IR, AO, PL. Wrote the paper: PL.

\section{Conflicts of Interest}

The authors declare no conflict of interest. 


\section{References}

1. Eming, S.A.; Krieg, T.; Davidson, J.M. Inflammation in wound repair: Molecular and cellular mechanisms. J. Investig. Dermatol. 2007, 127, 514-525.

2. Moura, L.I.; Dias, M.A.; Carvalho, E.; de Sousa, H.C. Recent advances on the development of wound dressings for diabetic foot ulcer treatment-A review. Acta Biomater. 2013, 9, 7093-7114.

3. Boateng, J.S.; Matthews, K.H.; Stevens, H.N.; Eccleston, G.M. Wound healing dressings and drug delivery systems: A review. J. Pharm. Sci. 2008, 97, 2892-2923.

4. Altinisik, A.; Yurdacoc, K. Synthesis, characterization, and enzymatic degradation of chitosan/PEG hydrogel films. J. Appl. Polym. Sci. 2011, 122, 1556-1563.

5. Gorgieva, S.; Kokol, V. Preparation, characterization, and in vitro enzymatic degradation of chitosan-gelatine hydrogel scaffolds as potential biomaterials. J. Biomed. Mater. Res. A 2012, 100, 1655-1667.

6. Tan, H.; Rubin, J.P.; Marra, K.G. Injectable in situ forming biodegradable chitosan-hyaluronic acid based hydrogels for adipose tissue regeneration. Organogenesis 2010, 6, 173-180.

7. Laurienzo, P. Marine polysaccharides in pharmaceutical applications: An overview. Mar. Drugs 2010, 8, 2435-2465.

8. Brachkova, M.I.; Marques, P.; Rocha, J.; Sepodes, B.; Duarte, M.A.; Pinto, J.F. Alginate films containing Lactobacillus plantarum as wound dressing for prevention of burn infection. J. Hosp. Infect. 2011, 79, 375-377.

9. Dantas, M.D.; Cavalcante, D.R.; Araujo, F.E.; Barretto, S.R.; Aciole, G.T.; Pinheiro, A.L.; Ribeiro, M.A.; Lima-Verde, I.B.; Melo, C.M.; Cardoso, J.C.; et al. Improvement of dermal burn healing by combining sodium alginate/chitosan-based films and low level laser therapy. J. Photochem. Photobiol. B 2011, 105, 51-59.

10. Peng, C.W.; Lin, H.Y.; Wang, H.W.; Wu, W.W. The influence of operating parameters on the drug release and anti-bacterial performances of alginate wound dressings prepared by three-dimensional plotting. Mater. Sci. Eng. C 2012, 32, 2491-2500.

11. Acarturk, F.; Takka, S. Calcium alginate microparticles for oral administration: II. Effect of formulation factors on drug release and drug entrapment efficiency. J. Microencapsul. 1999, 16, 291-301.

12. Kikuchi, A.; Kawabuchi, M.; Sugihara, M.; Sakurai, Y. Pulsed dextran release from calcium-alginate gel vedas. J. Control. Release 1997, 47, 21-29.

13. Lemoine, D.; Wauters, F.; Bouchend'homme, S.; Préat, V. Preparation and characterization of alginate microspheres coating a model antigen. Int. J. Pharm. 1998, 176, 9-19.

14. Murata, Y.; Meada, T.; Miyamoto, E.; Kawashima, S. Preparation of chitosan-reinforced alginate gel beads - effects of chitosan on gel matrix erosion. Int. J. Pharm. 1993, 96, 139-145.

15. Ravi Kumar, M.N.V.; Muzzarelli, R.A.A.; Muzzarelli, C.; Sashiwa, H.; Domb, A.J. Chitosan chemistry and pharmaceutical perspectives. Chem. Rev. 2004, 104, 6017-6084.

16. Dash, M.; Chiellini, F.; Ottenbrite, R.M.; Chiellini, E. Chitosan-A versatile semi-synthetyic polymer for biomedical applications. Prog. Polym. Sci. 2011, 36, 981-1014.

17. Hu, L.; Sun, Y.; Wu, Y. Advances in chitosan-based drug delivery vehicles. Nanoscale 2013, 5, 3103-3111. 
18. Patel, M.P.; Patel, R.R.; Patel, J.K. Chitosan mediated targeted drug delivery system: A review. J. Pharm. Sci. 2010, 13, 536-557.

19. Polk, A.; Amsden, B.; de Yao, K.; Peng, T.; Goosen, M.F.A. Controlled release of albumin from chitosan-Alginate microcapsules. J. Pharm. Sci. 1994, 83, 178-185.

20. Sezer, A.D.; Akbuga, J. Release characteristics of chitosan treated alginate beads: II. Sustained release of a low molecular drug from chitosan treated alginate beads. J. Microencapsul. 1999, 16, 678-696.

21. Sarmento, B.; Ribeiro, A.; Veiga, P.F.; Sampaio, P.; Neufeld, R.; Ferreira, D. Alginate/chitosan nanoparticles are effective for oral insulin delivery. Pharm. Res. 2007, 24, 2198-2206.

22. González-Rodríguez, M.L.; Holgado, M.A.; Sánchez-Lafuente, C.; Rabasco, A.M.; Fini, A. Alginate/chitosan particulate systems for sodium diclofenac release. Int. J. Pharm. 2002, 232, $225-234$.

23. Xu, Y.; Zhan, C.; Fan, L.; Wang, L.; Zheng, H. Preparation of dual crosslinked alginate-chitosan blend gel beads and in vitro controlled release in oral site-specific drug delivery system. Int. J. Pharm. 2007, 336, 329-337.

24. Lucinda-Silva, R.M.; Evangelista, M.C. Microspheres of alginate-chitosan containing isoziadin. J. Microencapsul. 2003, 20, 145-152.

25. World Health Organisation. Antimicrobial Resistance: Global Report on Surveillance; WHO Press: Geneva, Switzerland, 2014.

26. Cortivo, R.; Vindigni, V.; Iacobellis, L.; Abatangelo, G.; Pinton, P.; Zavan, B. Nanoscale particle therapies for wounds and ulcers. Nanomedicine 2010, 5, 641-656.

27. Goh, Y.F.; Shakir, I.; Hussain, R. Electrospun fibers for tissue engineering, drug delivery, and wound dressing. J. Mater. Sci. 2013, 48, 3027-3054.

28. Hussain, S.M.; Hess, K.L.; Gearhart, J.M.; Geiss, K.T.; Schlager, J.J. In vitro toxicity of nanoparticles in BRL 3A rat liver cells. Toxicol. In Vitro 2005, 19, 975-983.

29. Muzzarelli, R.A.A. Chitins and chitosans for the repair of wounded skin, nerve cartilage and bone. Carbohydr. Polym. 2009, 76, 167-182.

30. Kong, M.; Chen, X.G.; Xing, K.; Park, H.J. Antimicrobial properties of chitosan and mode of action: A state of the art review. Int. J. Food Microbiol. 2010, 144, 51-63.

31. Chung, Y.C.; Yeh, J.Y.; Tsai, C.F. Antibacterial characteristics and activity of water-soluble chitosan derivatives prepared by the Maillard reaction. Molecules 2011, 16, 8504-8514.

32. Kyzas, G.Z.; Bikiaris, D.N. Recent modifications of chitosan for adsorption applications: A critical and systematic review. Mar. Drugs 2015, 13, 312-337.

33. Signini, R.; Desbrières, J.; Campana Filho, S.P. On the stiffness of chitosan hydrochloride in acid-free aqueous solutions. Carbohydr. Polym. 2000, 43, 351-357.

34. Signini, R.; Campana Filho, S.P. On the preparation and characterization of chitosan hydrochloride. Polym. Bull. 1999, 42, 159-166.

35. Draget, K.I.; Oestgaard, K.; Smidsrod, O. Homogeneous alginate gels: A technical approach. Carbohydr. Polym. 1990, 14, 159-178.

36. Draget, K.I.; Skjak-Braek, G.; Smidsrod, O. Alginate based new materials. Int. J. Biol. Macromol. 1997, 21, 47-55. 
37. Catanzano, O.; Straccia, M.C.; Miro, A.; Ungaro, F.; Romano, I.; Mazzarella, G.; Santagata, G.; Quaglia, F.; Laurienzo, P.; Malinconico, M.; et al. Spray-by-Spray in situ cross-linking alginate hydrogels delivering a tea tree oil microemulsion. Eur. J. Pharm. Sci. 2015, 66, 20-28.

38. Alexander, B.R.; Murphy, K.E.; Gallagher, J.; Farrell, G.F.; Taggart, G. Gelation time, homogeneity, and rupture testing of alginate-calcium carbonate-hydrogen peroxide gels for use as wound dressings. J. Biomed. Mater. Res. B 2012, 100B, 425-431.

39. Del Nobile, M.A.; Laurienzo, P.; Malinconico, M.; Mensitieri, G.; Nicolais, L. New functionalized ethylene/vinyl alcohol co-polymers: Synthesis and water vapour transport properties. Packag. Technol. Sci. 1997, 10, 95-108.

40. Insan, N.G.; Payal, N.; Singh, M.; Yadav, A.; Chaudhary, B.; Srivastava, A. Post operative wound infection: Bacteriology and antibiotic sensitivity pattern. Int. J. Cur. Res. Rev. 2013, 5, 74-79.

41. Rossi, S.; Marciello, M.; Sandri, G.; Ferrari, F.; Bonferoni, M.C.; Papetti, A.; Caramella, C.; Dacarro, C.; Grisoli, P. Wound dressings based on chitosan and hyaluronic acid for the release of chlorhexidine diacetate in skin ulcer therapy. Pharm. Dev. Technol. 2007, 12, 415-422.

42. Oliva, A.; Passaro, I.; di Pasquale, A.; di Feo, A.; Criscuolo, M.; Zappia, V.; Della Ragione, F.; D’Amato, S.; Annunziata, M.; Guida, L. Ex vivo expansion of bone marrow stromal cells by platelet-rich plasma: A promising strategy in maxillo facial surgery. Int. J. Immunopathol. Pharmacol. 2006, 19, 47-53.

43. Amsterdam, D. Susceptibility testing of antimicrobials in liquid media. In Antibiotics in Laboratory Medicine, 4th ed.; Loman, V., Ed.; Williams \& Wilkins: Baltimore, MD, USA, 1996; pp. 52-111.

44. Wiegand, I.; Hilpert, K.; Hancock, R.E. Agar and broth dilution methods to determine the minimal inhibitory concentration (MIC) of antimicrobial substances. Nat. Protoc. 2008, 3, 163-175.

(C) 2015 by the authors; licensee MDPI, Basel, Switzerland. This article is an open access article distributed under the terms and conditions of the Creative Commons Attribution license (http://creativecommons.org/licenses/by/4.0/). 